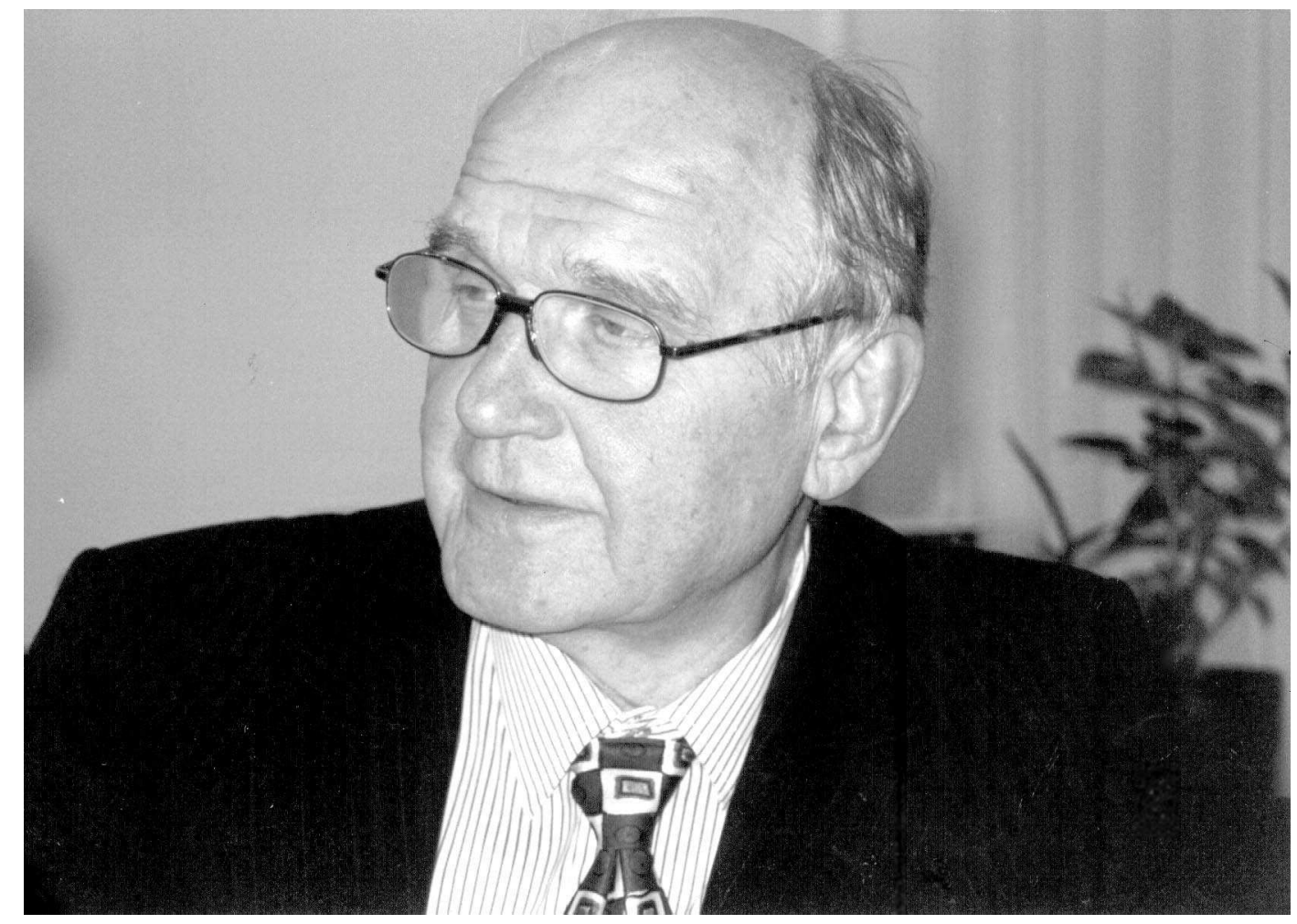

\title{
Celebration issues of the invited papers for Professor Ihor Yukhnovskii
}

On September 1, 2000 Academician, Professor Ihor Yukhnovskii, a prominent Ukrainian scientist and a founder of the Lviv school of statistical physics, will celebrate his 75th birthday. On this occasion, Institute for Condensed Matter Physics and Editorial board of "Condensed Matter Physics" decided to honour him with a collection of scientific papers. With great pleasure numerous researchers, colleagues and coworkers of Ihor Yukhnovskii contribute their articles. Editorial board of "Condensed Matter Physics" express sincere gratitude to them. 
The professional contributions broadly cover the fields of general problems of statistical physics, theory of superfluidity and Bose condensation, non-equilibrium statistics, theory of phase transitions and critical phenomena, theory of liquid state, soft matter and plasmas, statistical theory of metal alloys, strongly correlated electron systems and solid state physics. All the papers are connected in one or another way to scientific interest and work of Professor Ihor Yukhnovskii during his productive and successful scientific life. Among the papers there are several devoted to the problems which are of Ihor Yukhnovskii's interest only of late, such as the problems of catalysis, mathematical economics and physical processes in the "Shelter" (Chornobyl).

This issue contains the first part of the papers contributed. The continuation will be given in the next number of "Condensed Matter Physics".

The presented collection of papers demonstrate that the research fields, associated with Professor Ihor Yukhnovskii's activity, are being successfully developed in Ukraine as well as in the world. His numerous pupils and followers make an important contribution to the further development of these fields of theoretical physics. 